\title{
El acceso vascular
}

\author{
Vascular Access \\ José Horacio Cano-Cervantes* \\ * Departamento de Nefrología y Unidad de Trasplante Renal. Centro Médico Nacional «20 de Noviembre», ISSSTE.
}

- Todo paciente que haya alcanzado una tasa de filtración glomerular (TFG) $<30 \mathrm{~mL} / \mathrm{min}$ deberá ser referido y orientado en la elección del acceso vascular (AV).

- La morbilidad y mortalidad tienen una relación directa con el tipo de AV. Cuando se usa un catéter temporal en comparación con uno definitivo, se incrementa cuatro veces el riesgo de complicaciones infecciosas.

- Existen tres tipos de accesos vasculares: los primeros son los catéteres venosos centrales (CVC) temporales no tunelizados (duración no mayor de cuatro semanas) y tunelizados; en segundo lugar, está la fístula arteriovenosa (FAV) con un proceso de maduración por lo menos de tres meses, y en tercer lugar, los injertos o protésicos, cuya colocación es por lo menos de tres a seis semanas previas al inicio de la hemodiálisis. Las guías internacionales sugieren siempre a la FAV como primera opción y al CVC como la última.

- El trasplante renal puede priorizarse en el paciente sin opciones de reemplazo renal cuando se presente pérdida de cavidad abdominal y agotamiento de accesos vasculares, o bien disfunción del angioacceso $(\mathrm{Qb}<300 \mathrm{~mL} / \mathrm{h}$ y $\mathrm{PA}>-250 \mathrm{mmHg}$ y $\mathrm{Kt} / \mathrm{V}<1.2)$ demostrable en cada centro hospitalario y uso de catéteres translumbares o intracavitarios.

Cuando el paciente ha alcanzado el estadio 4 de enfermedad renal crónica (ERC) (TFG $30 \mathrm{~mL}$ / $\mathrm{min} / 1.73 \mathrm{~m}^{2} \mathrm{SC}$ ) o se considere un rápido deterioro de la función renal, definido como la reducción de la tasa de filtración glomerular (TFG) $>5 \mathrm{~mL} /$ $\min / 1.72 \mathrm{~m}^{2} \mathrm{SC}$ o $>10 \mathrm{~mL} / \mathrm{min} / 1.73 \mathrm{~m}^{2} \mathrm{SC}$ por cinco años, idealmente, se deberá orientar al paciente en la elección del acceso vascular. ${ }^{1}$ En México, se desconoce la incidencia y prevalencia de la ERC, por lo tanto, se desconoce cuál es la prevalencia de la terapia de reemplazo renal (TRR).

En nuestro país, prevalece la diálisis peritoneal (DP) como terapia de reemplazo renal. No obstante, en los últimos años, se ha ido incrementando la proporción de individuos tratados con hemodiálisis (HD). Un ejemplo de esto es el registro llevado a cabo en el estado de Jalisco, el cual reportó que la proporción de pacientes con DP ha disminuido de forma considerable a un $44.8 \% .^{2}$ Según Méndez-Durán, en diciembre de 2014, se registraron 55,101 pacientes al IMSS; sin embargo, de éstos, $58 \%$ necesitaba DP y $42 \% \mathrm{HD}^{3}$

El acceso vascular (AV) es el punto anatómico por donde se accederá al torrente sanguíneo del enfermo renal y es el mismo por donde se extraerá y retornará la sangre una vez que ha pasado por el circuito extracorpóreo de depuración extrarrenal. ${ }^{4}$

Existen tres tipos de accesos vasculares:

1. Temporales. Es un catéter venoso central (CVC) que se coloca entre una vena con el calibre necesario (habitualmente, venas yugulares, subclavias o femorales) para poder proporcionar flujos sanguíneos suficientes para la realización de la TRR; cuando debe ser utilizado durante un periodo de tiempo superior a las 2 o 4 semanas, se tuneliza subcutáneamente para evitar infecciones. 
2. Fístula arteriovenosa (FAV). Consiste en la conexión de una arteria con una vena a través de una anastomosis término-lateral o latero-lateral. El objetivo es que la vena se arterialice para poder proceder a su punción con facilidad y para que proporcione el suficiente flujo sanguíneo para la hemodiálisis. Dado que la FAV requiere de tiempo para su punción o proceso de «maduración», las recomendaciones implementadas por KDOQI (por sus siglas en inglés Kidney Disease Outcomes Quality Initiative) de $2015^{1}$ sugieren que debe enviarse al paciente con TFG $<25 \mathrm{~mL} / \mathrm{min} / 1.73 \mathrm{~m}^{2} \mathrm{SC}$ o creatinina sérica $>4 \mathrm{mg} / \mathrm{dL}$ (o progresión rápida, la cual, de manera breve, se describió al inicio del presente capítulo); todo esto es con la finalidad de un proceso de maduración de por lo menos tres meses.

3. AV protésico o injerto. Consiste en la colocación de un fragmento de politetrafluoroetileno (PTFE) entre una arteria y una vena. Este injerto será el fragmento canulable del AV. Las recomendaciones por KDOQI de $2015^{1}$ sugieren su colocación por lo menos de tres a seis semanas previas al inicio de la hemodiálisis; su principal recomendación se centra en pacientes con duración de TRR menor de dos años. Los dispositivos $\mathrm{HeRO}^{\circledR}$ son considerados por la Food and Drug Administration como un injerto; sus indicaciones de uso son: falla de FAV o injerto con necesidad de múltiples intervenciones, $\mathrm{Kt} / \mathrm{V}<1.4$ de manera recurrente y dependencia de catéteres por infecciones recurrentes.

Por un lado, la morbilidad y mortalidad tiene una relación directa con el tipo de AV. Por el otro, el riesgo de complicaciones infecciosas incrementa cuatro veces cuando se usa un catéter temporal en comparación con uno definitivo. ${ }^{5}$ El estudio CHOICE mostró RR de mortalidad de 1.47 de CVC en comparación con FAV. ${ }^{6}$ Por su parte, el análisis de Ravani y colaboradores ${ }^{7}$ concluye que el CVC se asocia con mayor mortalidad con RR 1.53 (IC95\%1.41-1.67), infecciones fatales con RR 2.12 (IC95\%1.79-2.52) y eventos cardiovasculares con RR 1.38 (IC95\% 1.24-1.54) comparados con aquellos pacientes que usaron FAV.

El acceso vascular ideal deberá cumplir con ciertas características: ${ }^{8-10}$

- Anatomía. Es necesario que tenga una adecuada calidad de vasos para correctos flujos sanguíneos y adecuada prescripción. Dicho esto, también es necesario considerar la calidad de vasos en diabé- ticos, personas de la tercera edad y mujeres con diabetes mellitus.

- Menor probabilidad de falla primaria. Esto se presenta en $47-60 \%$ para FAV en relación con la construcción del $\mathrm{AV}$, siendo mayor en braquiocefálicas (32\%) y menores en las que se realizan en la porción más proximal del miembro torácico superior (15\%); en injertos, la falla primaria suele presentarse en el $20 \%$.

- Funcionalidad. Sin ser superior la FAV en comparación con los injertos a dos años, y finalmente, hay menor supervivencia en CVC.

- Complicaciones. La mayor parte de ellas están presentes en CVC (permanentes) al año de su colocación; la disfunción de angioacceso se define como: $\mathrm{Qb}<300 \mathrm{~mL} / \mathrm{min}$ y presión de lumen arterial $>-250 \mathrm{mmHg}(15 \%)$, infecciosas: 9\%, mecánicas: estenosis (2\%).

- Preferencia del paciente. Deberá considerarse la decisión del paciente en la colocación del AV; además se deberá explicar ampliamente los riesgos y beneficios de cada uno de ellos.

Las guías de accesos vasculares publicadas por KDOQI (2015), ${ }^{1}$ las de la Canadian Society of Nephrology ${ }^{11}$ (2006), la European Renal Best Practice ${ }^{12}$ (ERBP; 2019) y la Kidney Health Australia-Caring for Australasians with Renal Impairment ${ }^{13}$ (KHA-CARl; 2013) recomiendan:

- Las FAV son preferidas como acceso vascular inicial. Las guías KDOQI y ERBP recomiendan las fístulas radiocefálicas como primera opción, seguidas por las braquiocefálicas y, como tercera opción, las braquiobasílicas.

- Los injertos son recomendados en aquellos pacientes que poseen vasos sanguíneos inadecuados o en donde la duración de la TRR sea menor a dos años.

- Los catéteres serán considerados como última opción de acceso.

En México, las recomendaciones emitidas por las guías de práctica clínica del Centro Nacional de Excelencia Tecnológica en Salud (CENETEC) ${ }^{14}$ hacen referencia a la FAV como primera opción y al nefrólogo y/o personal médico capacitado como el responsable de la colocación del acceso vascular.

\section{CON CUÁL INICIAR: RECOMENDACIONES}

Todo paciente antes de diálisis, cuya expectativa de inicio de diálisis sea a un año y/o inicio urgente mediante CVC: 
1) Deberá ser enviado a la creación de una fístula arteriovenosa, excepto:

- Si la expectativa de vida es limitada por: cáncer metastásico, falla cardiaca severa, edad avanzada o cualquier condición que a consideración del médico tratante limite su esperanza de vida.

- Si la expectativa de hemodiálisis es corta, sobre todo en trasplante renal de donación viva.

- Si la duración esperada en TRR es < 2 años, se recomienda el uso de injerto.

2) En catéteres venosos centrales se debe usar:

- Catéter permanente sobre temporal.

- Catéter permanente tunelizado sobre injerto en paciente con protocolo de donación con posibilidad de trasplante menor a dos años.

- Terapia puente en el proceso de maduración de una FAV.

3) Agotamiento de angioaccesos superiores, considerar diálisis peritoneal y trasplante renal antes de accesos vasculares inferiores.

4) En caso de que el receptor tenga un acceso vascular al momento del trasplante, se sugiere:

- Usar el acceso temporal (CVC) o permanente durante el trasplante, siempre y cuando esté libre de infecciones y su colocación permita un adecuado flujo de soluciones y administración de medicamentos.

- Colocar el CVC en el lado contralateral, preferentemente mediante guía ultrasonográfica, en caso de ser portador de FAV o injerto.

\section{A QUIÉN PRIORIZAR}

La elección del paciente candidato a trasplante renal con agotamiento de accesos vasculares no posee un grado de recomendación en la mayoría de las guías internacionales. No obstante, la guía $B C$ Transplant ${ }^{15}$ hace mención como prioridad 2 en la elección del receptor únicamente cuando es precedido de un paciente altamente sensibilizado.

Por tanto, nuestra recomendación en priorizar al paciente con agotamiento de accesos vasculares se puede resumir en la conjunción de dos puntos:

1. La disfunción del angioacceso $(\mathrm{Qb}<300 \mathrm{~mL} / \mathrm{h}$ y $\mathrm{PA}>-250 \mathrm{mmHg}$ y $\mathrm{Kt} / \mathrm{V}<1.2)$ con evidencia demostrable de cada centro hospitalario.

2. El uso de catéteres translumbares o intracavitarios.

Finalmente, debe hacerse hincapié que, pese a tratarse de un acceso vascular translumbar o intraca- vitario, éste no deberá considerarse prioridad si no se documenta ninguna disfunción.

\section{REFERENCIAS}

1. National Kidney Foundation. KDOQI Clinical Practice Guideline for Hemodialysis Adequacy: 2015 Update. Am J Kidney Dis. 2015; 66 (5): 884-930.

2. González-Bedat MC, Rosa-Diez G, Fernández-Cean JM, Ordúñez P, Ferreiro A, Douthat W. Los registros nacionales de diálisis y trasplante renal en América Latina: cómo implementarlos y mejorarlos. Rev Panam Salud Publica. 2015; 38 (3): 254-260.

3. Méndez-Durán A, Ignorosa-Luna MH, Pérez-Aguilar G, GonzálezIzquierdo JJ, Dávila-Torrese J. Estado actual de las terapias sustitutivas de la función renal en el Instituto Mexicano del Seguro Social. Rev Med Inst Mex Seguro Soc. 2016; 54 (5): 588-593.

4. González MA, Martínez R. Capítulo 1: Definición del acceso vascular. En: Manual de accesos vasculares para hemodiálisis. Sociedad Española de Diálisis. Ed. Marge Médica Books; 2010. pp. 27-30.

5. Lok CE, Foley R. Vascular access morbidity and mortality: Trends of the last decade. Clin J Am Soc Nephrol. 2013; 8 (7): 1213-1219.

6. Astor BC, Eustace JA, Powe NR, Klag MJ, Fink NE, Coresh J. Type of vascular access and survival among incident hemodialysis patients: the choices for healthy outcomes in caring for ESRD (CHOICE) study. J Am Soc Nephrol. 2005; 16 (5): 1449-1455.

7. Ravani P, Palmer SC, Oliver MJ, Quinn RR, MacRae JM, Tai DJ et al. Associations between hemodialysis access type and clinical outcomes: a systematic review. J Am Soc Nephrol. 2013; 24 (3): 465-473.

8. Poinen K, Quinn RR, Clarke A, Ravani P, Hiremath S, Miller LM et al. Complications from tunneled hemodialysis catheters: a canadian observational cohort study. Am J Kidney Dis. 2019; 73 (4): 467-475.

9. Cheung AK, Imrey PB, Alpers CE, Robbin ML, Radeva M, Larive $B$ et al. Intimal hyperplasia, stenosis, and arteriovenous fistula maturation failure in the hemodialysis fistula maturation study. $\mathrm{J}$ Am Soc Nephrol. 2017; 28 (10): 3005-3013.

10. Drew DA, Lok CE, Cohen JT, Wagner M, Tangri N, Weiner DE. Vascular access choice in incident hemodialysis patients: a decision analysis. J Am Soc Nephrol. 2015; 26 (1): 183-191.

11. Jindal K, Chan CT, Deziel C, Hirsch D, Soroka SD, Tonelli M et al. Hemodialysis clinical practice guidelines for the Canadian Society of Nephrology. J Am Soc Nephrol. 2006; 17 (3 Suppl 1): S1-27.

12. ERBP Guideline Development Group on Vascular Access. Clinical Practice Guideline on peri- and postoperative care of arteriovenous fistulas and grafts for haemodialysis in adults. Nephrol Dial Transplant. 2019; 34 Suppl 2: ii1-ii42.

13. Polkinghorne KR, Chin GK, MacGinley RJ, Owen AR, Russell C, Talaulikar GS et al. KHA-CARI Guideline: Vascular access central venous catheters, arteriovenous fistulae and arteriovenous grafts. Nephrology (Carlton). 2013; 18 (11): 701-705.

14. Manejo de accesos vasculares en hemodiálisis en adultos con insuficiencia renal crónica en el segundo y tercer nivel de atención. Guía de práctica clínica; CENETEC. ISSSTE-680-13.

15. Clinical guidelines for kidney transplantation. Canadian Society of Transplantation consensus guidelines on eligibility for kidney transplantation. BC Transplant. 2018; 1-48.

Correspondencia:

Dr. José Horacio Cano-Cervantes

E-mail:drkno@hotmail.es 